\title{
Polynomial realizations of some combinatorial Hopf algebras
}

\author{
Loïc Foissy, Jean-Christophe Novelli and Jean-Yves Thibon*
}

\begin{abstract}
We construct explicit polynomial realizations of some combinatorial Hopf algebras based on various kinds of trees or forests, and some more general classes of graphs, ranging from the Connes-Kreimer algebra to an algebra of labelled forests isomorphic to the Hopf algebra of parking functions and to a new noncommutative algebra based on endofunctions admitting many interesting subalgebras and quotients.
\end{abstract}

Mathematics Subject Classification (2010). 05C05; 16W30.

Keywords. Hopf algebras of decorated rooted trees, free quasi-symmetric functions, parking functions.

\section{Introduction}

One knows many examples of Hopf algebras based on various kinds of trees or forests [3], [6], [7], [20], [16], [17], [24]. Such algebras are increasingly popular, mainly because of their applications to renormalization problems in quantum field theory [19], [3], but some of them occurred earlier in combinatorics [12], [13] or in numerical analysis [14].

The simplest one, generally known as the Connes-Kreimer algebra [3], is a commutative algebra freely generated by rooted trees, endowed with a coproduct defined in terms of admissible cuts.

This is a basic example of a combinatorial Hopf algebra, a heuristic notion encompassing a large class of graded connected Hopf algebras based on combinatorial objects, endowed with some extra structure such as distinguished bases, scalar products or degree-preserving products (called internal products). A distinctive feature of combinatorial Hopf algebras is that products and coproducts in distinguished bases are given by combinatorial algorithms. However, in many cases, the basis elements can be realized as polynomials ${ }^{1}$ (commutative or not) in some auxiliary set of variables, in such a way that the product of the algebra becomes the usual product of

\footnotetext{
*Partially supported by a PEPS project of the CNRS.

${ }^{1}$ By polynomials in infinitely many variables we actually mean elements of an inverse limit of polynomial algebras in the category of graded algebras. That is, a polynomial can have infinitely many monomials, but must be of bounded degree.
} 
polynomials, and the coproduct a simple trick of "doubling the variables" (see, e.g., [4], [25], [24] for detailed examples).

Such a construction was not known for the Connes-Kreimer algebra, despite the fact that it is one of the simplest examples. The present paper provides such a construction, which in turn will be obtained by specialization of a new realization of a Hopf algebra of labelled forests [10], itself isomorphic to the (dual) Hopf algebra of parking functions [25]. This provides as well realizations of the noncommutative Connes-Kreimer algebra (isomorphic to the Loday-Ronco algebra of planar binary trees) [6], [7], [20] and new morphisms between these algebras and other combinatorial Hopf algebras.

Previously known realizations are defined in terms of an auxiliary alphabet $A$, endowed with some ordering. A given combinatorial Hopf algebra is then realized by interpreting the elements of some basis as the sum of all words over $A$ sharing some specific property (e.g., descent set, standardization, packing, parkization), the product being then the ordinary product of polynomials, and the coproduct being the ordinal sum $A+B$ of two isomorphic copies of the ordered set $A$.

As we shall see, it is possible to extend this approach to the algebras of the ConnesKreimer family, provided that one replaces the order on $A$ by another kind of binary relation, for which an analog of the ordinal sum can be defined. Actually, this kind of construction works for a slightly more general class of algebras. Our starting point is an algebra of ordered forests, where edges are oriented towards the roots and a loop is added on each root. This amounts to interpreting ordered forests as a special class of endofunctions (acyclic endofunctions, those for which the graph with edges $i \rightarrow j$ for $j=f(i)$ has no cycle of length greater than 1). At the opposite extreme, the graphs representing permutations consist only of cycles. A similar but different construction can be given for this case, and one obtains in this way a realization of the dual of the commutative Hopf algebra of permutations of [18]. Finally, on can again modify the construction so as to obtain a realization of a new Hopf algebra based on all endofunctions. This last one admits many interesting subalgebras and quotients, including the previously discussed ones, for which the construction provides different realizations.

\section{Rooted trees and rooted forests}

Throughout the paper $\mathbb{K}$ will denote a field of characteristic zero.

2.1. Reminders on rooted trees and forests. A rooted tree is a finite tree with a distinguished vertex called the root. A rooted forest is a finite graph $\mathcal{F}$ such that any connected component of $\mathscr{F}$ is a rooted tree. The set of vertices of the rooted forest $\mathscr{F}$ is denoted by $V(\mathscr{F})$.

Let $\mathcal{F}$ be a rooted forest. The edges of $\mathscr{F}$ are oriented downwards (from the leaves to the roots). If $v, w \in V(\mathcal{F})$, with $v \neq w$, we shall write $v \rightarrow w$ if there is 
an edge in $\mathcal{F}$ from $v$ to $w$, and $v \rightarrow w$ if there is an oriented path from $v$ to $w$ in $\mathscr{F}$.

Let $v$ be a subset of $V(\mathscr{F})$. We shall say that $v$ is an admissible cut of $\mathscr{F}$, and we shall write $v \models V(\mathscr{F})$ if $v$ is totally disconnected, that is to say that there is no path from $v$ to $w$ in $\mathscr{F}$ for any pair $(v, w)$ of distinct elements of $\boldsymbol{v}$. If $\boldsymbol{v} \models V(\mathcal{F})$, we denote by Lea $v$ F the rooted subforest of $\mathscr{F}$ obtained by keeping only the vertices above $\boldsymbol{v}$, that is to say $\{w \in V(\mathscr{F}) \mid$ there exists $v \in \boldsymbol{v}$ with $w \rightarrow v\} \cup \boldsymbol{v}$. We denote by $\operatorname{Roo}_{v} \mathcal{F}$ the rooted subforest obtained by keeping the other vertices.

2.2. The Connes-Kreimer Hopf algebras. Connes and Kreimer proved in [3] that the vector space $\mathbf{H}$ spanned by rooted forests can be turned into a Hopf algebra. Its product is given by the disjoint union of rooted forests, and the coproduct is defined for any rooted forest $\mathcal{F}$ by

$$
\Delta(\mathcal{F})=\sum_{v \models V(\mathcal{F})} \operatorname{Roo}_{\boldsymbol{v}} \mathcal{F} \otimes \operatorname{Lea}_{\boldsymbol{v}} \mathcal{F} .
$$

For example,

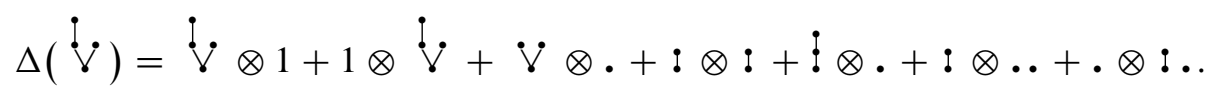

This Hopf algebra is commutative and noncocommutative. Its dual is the universal enveloping algebra of the free pre-Lie algebra on one generator [2].

A similar construction can be done on plane forests. The resulting noncommutative, noncocommutative Hopf algebra $\mathbf{H}_{\mathrm{NCK}}$ is called the noncommutative ConnesKreimer Hopf algebra [6], [7]. It is isomorphic to the Hopf algebra of planar binary trees [20].

\section{Ordered rooted trees and permutations}

We recall here a generalization of the construction of the product and the coproduct of $\mathbf{H}$ to the space spanned by ordered rooted forests introduced in [10].

3.1. Hopf algebra of ordered trees. An ordered (rooted) forest is a rooted forest with a total order on the set of its vertices. The set of ordered forests will be denoted by $\mathbf{F}_{o}$; for all $n \geq 0$, the set of ordered forests with $n$ vertices will be denoted by $\mathbf{F}_{o}(n)$. An ordered (rooted) tree is a connected ordered forest. The set of ordered trees will be denoted by $\mathbf{T}_{o}$; for all $n \geq 1$, the set of ordered trees with $n$ vertices will be denoted by $\mathbf{T}_{o}(n)$. The $\mathbb{K}$-vector space generated by $\mathbf{F}_{o}$ is denoted by $\mathbf{H}_{o}$. It is a graded space, the homogeneous component of degree $n$ being $\operatorname{Vect}\left(\mathbf{F}_{o}(n)\right)$ for all $n \in \mathbb{N}$. 
For example,

$$
\begin{aligned}
& \mathbf{T}_{o}(1)=\left\{\bullet_{1}\right\}, \\
& \mathbf{T}_{o}(2)=\left\{\mathbf{b}_{1}^{2},:_{2}^{1}\right\} \text {, }
\end{aligned}
$$

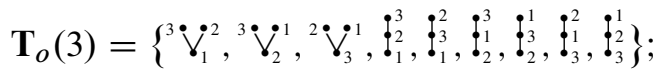

and

$$
\begin{aligned}
& \mathbf{F}_{o}(0)=\{1\}, \\
& \mathbf{F}_{o}(1)=\left\{\bullet_{1}\right\}, \\
& \mathbf{F}_{o}(2)=\left\{\bullet_{1} \cdot \bullet_{2}, \mathfrak{d}_{1}^{2}, \mathfrak{l}_{2}^{1}\right\} \text {, }
\end{aligned}
$$

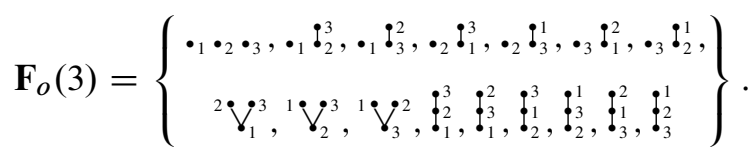

If $\mathcal{F}$ and $\mathscr{E}$ are two ordered forests, then the rooted forest $\mathcal{F} \mathscr{E}$ is seen as the ordered forest such that, for all $v \in V(\mathcal{F}), w \in V(\mathscr{E}), v<w$. This defines a noncommutative product on the set of ordered forests. For example, the product of $\cdot_{1}$ and $:_{1}^{2}$ gives.$_{1} l_{2}^{3}$, whereas the product of $:_{1}^{2}$ and $\bullet_{1}$ gives $:_{1}^{2} \cdot_{3}=._{3}:_{1}^{2}$. This product is linearly extended to $\mathbf{H}_{o}$, which in this way becomes a graded algebra.

The number of ordered forests with $n$ vertices is $(n+1)^{n-1}$, which is also the number of parking functions of length $n$. By definition, $\mathbf{H}_{o}$ is free over irreducible ordered forests (that is to say ordered forests which cannot be written as the product of two nonempty ordered forests), which are in bijection with connected parking functions. For example, here are the connected ordered forests with $k \leq 3$ vertices:

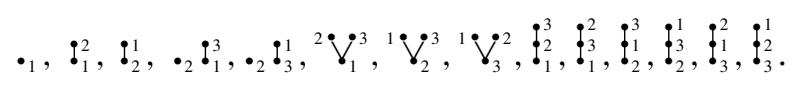

Hence, as an associative algebra $\mathbf{H}_{o}$ is isomorphic to the Hopf algebra of parking functions PQSym introduced in [25].

If $\mathcal{F}$ is an ordered forest, then any subforest of $\mathcal{F}$ is also ordered. In [10], a coproduct $\Delta: \mathbf{H}_{o} \mapsto \mathbf{H}_{o} \otimes \mathbf{H}_{o}$ on $\mathbf{H}_{o}$ has been defined in the following way: for all $\mathscr{F} \in \mathbf{F}_{o}$,

$$
\Delta(\mathcal{F})=\sum_{v \models V(\mathcal{F})} \operatorname{Roo}_{\boldsymbol{v}} \mathcal{F} \otimes \operatorname{Lea}_{\boldsymbol{v}} \mathcal{F} .
$$

As for the Connes-Kreimer Hopf algebra of rooted trees [3], one can prove that this coproduct is coassociative, so $\mathbf{H}_{o}$ is a graded Hopf algebra. For example,

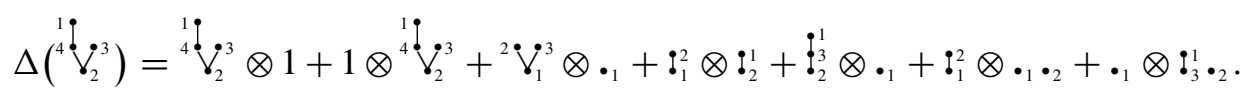

Theorem 3.1. As a Hopf algebra, $\mathbf{H}_{o}$ is isomorphic to the graded dual PQSym* of PQSym. 
Note. Actually, PQSym is self-dual, but as we shall see, $\mathbf{H}_{o}$ admits WQSym as a natural quotient rather than as a natural subalgebra, which is also the case of PQSym* .

Proof. We shall only give here the main ideas of the proof, see [9] for more details. Another product, denoted by $\nwarrow$, is defined on the augmentation ideal of $\mathbf{H}_{o}$ : if $\mathscr{F}$ and $\mathcal{E}$ are two ordered forests, $\mathscr{F} \nwarrow \mathscr{E}$ is the ordered forest obtained by grafting $\mathscr{E}$ shifted by the number of vertices of $\mathcal{F}$ on the greatest vertex of $\mathscr{F}$. For example,

$$
:_{1}^{2} \nwarrow \cdot \cdot_{1} \cdot \bullet_{2}={ }^{3} \dddot{Y}_{1}^{4} \quad \text { and }:_{2}^{1} \nwarrow \cdot_{1} \cdot \bullet_{2}={ }^{1} \stackrel{3}{Y}_{2}^{4} \text {. }
$$

This product is associative, and satisfies a certain compatibility with the product of $\mathbf{H}_{o}$. The coproduct of $\mathbf{H}_{o}$ also splits into two parts, separating the admissible cuts, according to whether the greatest vertex of $\mathcal{F}$ is in $\operatorname{Roo}_{\boldsymbol{v}} \mathcal{F}$ or Lea $\boldsymbol{F}$ F. These coproducts make the augmentation ideal of $\mathbf{H}_{o}$ a dendriform coalgebra, and there is a certain compatibility (called duplicial) between each product and each coproduct of $\mathbf{H}_{o}$, making $\mathbf{H}_{o}$ what is called in [9] a Dup-Dend bialgebra. Moreover, the Hopf algebra PQSym* is a Dup-Dend bialgebra. A rigidity theorem, similar with the rigidity theorem for bidendriform bialgebra of [8], tells then that a graded, connected Dup-Dend bialgebra is free. As a consequence, as $\mathbf{H}_{o}$ and $\mathbf{P Q S y m}{ }^{*}$ have the same Poincaré-Hilbert series, they are isomorphic as graded Dup-Dend bialgebras, hence also as graded Hopf algebras [9].

3.2. A realization of $\mathbf{H}_{\boldsymbol{o}}$. The Hopf algebra of ordered rooted forests can be realized by explicit polynomials in an auxiliary alphabet of bi-indexed variables

$$
A=\left\{a_{i j} \mid 1 \leq i \leq j\right\} .
$$

On such an alphabet, we consider the relation $\prec$ defined by

$$
a_{i j} \prec a_{j k} \quad \text { for } i \leq j \text { and } j<k .
$$

In particular, for all $i \leq j, a_{i j} \prec a_{i j}$ if and only if $i=j$, so that this relation is neither reflexive nor transitive. We call the pair $(A, \prec)$ a $\prec$-alphabet. This is an analog of the notion of ordered alphabet used for most other combinatorial Hopf algebras. If $(B, \prec)$ is another $\prec$-alphabet, their $\prec$-sum $A \oplus B$ is defined as their disjoint union endowed with the $\prec$-relation restricting to the original ones of $A$ and $B$ and such that

$$
a_{i j} \prec b_{k k} \quad \text { for all } i \leq j \text { and all } k .
$$

Let $\mathcal{F}$ be an ordered forest with $n$ vertices. We attach to the root of each tree of $\mathcal{F}$ a loop, that is to say an edge from the root to itself. For example, we shall consider

$$
{ }^{3} \mathfrak{V}_{1}^{4} \text { as } \quad{ }^{3} \mathfrak{J}_{1}^{4} \text {. }
$$


There is then a natural bijection from the set of edges of $\mathcal{F}$ (including the edges of the loops) and the vertices of $\mathscr{F}$, associating with an edge of $\mathscr{F}$ its initial vertex. As the set of vertices of $\mathscr{F}$ is totally ordered, the set of edges of $\mathscr{F}$ is also totally ordered by means of the bijection. We shall denote by $e_{1}<\cdots<e_{n}$ the set of edges of $\mathcal{F}$.

Let $w=w_{1} \ldots w_{n}$ be a word of length $n$ over $A$. We say that $w$ is $\mathscr{F}$-compatible if the following holds: if $k, l \in\{1, \ldots, n\}$ are such that the initial vertex of $e_{k}$ is the terminal vertex of $e_{l}$ (or, equivalently, if $l \rightarrow k$ in $\mathscr{F}$ ), then $w_{k} \prec w_{l}$. We write $w \vdash \mathscr{F}$. Now define the polynomials

$$
S^{\mathscr{F}}(A)=\sum_{w \vdash \mathscr{F}} w .
$$

For example, let

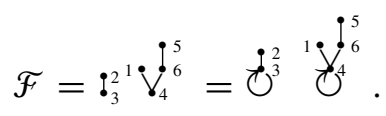

Then

$$
S^{\mathcal{F}}=\sum_{\substack{w_{3}<w_{2}, w_{3} \\ w_{4} \\ w_{4} w_{1}, w_{4}, w_{6} \\ w_{6} \prec w_{5}}} w_{1} w_{2} w_{3} w_{4} w_{5} w_{6}=\sum_{\substack{i_{3}<i_{2} \\ i_{4}<i_{1}, i_{6} \\ i_{6}<i_{5}}} a_{i_{4} i_{1}} a_{i_{3} i_{2}} a_{i_{3} i_{3}} a_{i_{4} i_{4}} a_{i_{6}} i_{5} a_{i_{4} i_{6}} .
$$

Theorem 3.2. The polynomials $S^{\mathscr{F}}(A)$ provide a faithful realization of $\mathbf{H}_{o}$, that is,

$$
S^{\mathcal{F}} S^{\mathscr{E}}=S^{\mathcal{F} \mathscr{E}} .
$$

If we allow $A$ and $B$ to commute and identify $P(A) Q(B)$ with $P \otimes Q$, then

$$
S^{\mathcal{F}}(A \oplus B)=\sum_{v \models V(\mathcal{F})} S^{\mathrm{Roo}_{v} \mathcal{F}}(A) S^{\text {Lea }_{v} \mathcal{F}}(B)=S^{\Delta(\mathcal{F})} .
$$

Proof. Let us first prove the product rule. Let $\mathcal{F}$ be an ordered forest with $k$ vertices and $\mathscr{E}$ be an ordered forest with $l$ vertices. In the ordered forest $\mathcal{F} \mathscr{G}$, the vertices of $\mathcal{F}$ are the first $k$ vertices, the vertices of $\mathscr{E}$ are the last $l$ ones, and there are no edges between the vertices of $\mathcal{F}$ and the vertices of $\mathscr{G}$. Consequently, a word $w_{1} \ldots w_{k+l}$ is $\mathcal{F} \mathscr{E}$-compatible if and only if $w_{1} \ldots w_{k}$ is $\mathscr{F}$-compatible and $w_{k+1} \ldots w_{k+l}$ is $\mathscr{G}$-compatible. Hence

$$
S^{\mathcal{F} \mathscr{E}}=\sum_{w^{\prime} \models \mathscr{F}, w^{\prime \prime} \models \mathscr{E}} w^{\prime} w^{\prime \prime}=S^{\mathscr{F}} S^{\mathscr{E}} .
$$

Let us now prove that the realization is faithful. Let $w=a_{i_{1}, j_{1}} \ldots a_{i_{n}, j_{n}}$ be a word on the alphabet $A$. Let $J(w)=\left\{j_{1}, \ldots, j_{n}\right\}$ and $j(w)=\operatorname{card}(J(w))$. Then $j(w)$ defines a degree on $\mathbb{K}\langle\langle A\rangle\rangle$. If $P=\sum x_{w} w$ is an element of $\mathbb{K}\langle\langle A\rangle\rangle$, we denote by $\widetilde{P}$ the component of $P$ of maximal $J(w)$, if it exists. In particular, if the degree of the words appearing in $P$ is bounded, then $\widetilde{P}$ exists. 
Let $\mathcal{F}$ be an ordered forest. The degree of the words $w$ appearing in $S^{\mathscr{F}}(A)$ is the number $n$ of vertices of $\mathscr{F}$, so $\widetilde{S}^{\mathscr{F}}(A)$ exists. As there are $\mathscr{F}$-compatible words $w$ such that $j(w)=n, j\left(S^{\mathcal{F}}(A)\right)=n$. Hence, if $w=a_{i_{1}, j_{1}} \ldots a_{i_{n}, j_{n}}$ appears in $\tilde{S}^{\mathscr{F}}$, then necessarily $j_{1}, \ldots, j_{n}$ are all distinct, so we can reconstruct $\mathscr{F}$ from $w$ : the vertex $k$ is a root if and only if $i_{k}=j_{k}$ and there is an edge from the vertex $k$ to the vertex $l$ in $\mathscr{F}$ if and only if $i_{k}=j_{l}$. As a consequence, the $\widetilde{S}^{\mathcal{F}}(A)$ are linearly independent. And so are the $S^{\mathscr{F}}(A)$.

Consider now a word $w=c_{i_{1} j_{1}} \ldots c_{i_{n} j_{n}}$ in $S^{\mathscr{F}}(A \oplus B)$. If $c_{i_{k} j_{k}}$ belongs to $B$, and if $l \rightarrow k$ in $\mathscr{F}$, then $c_{i_{k} j_{k}} \prec c_{i_{l} j_{l}}$, so $c_{i_{l} j_{l}}$ also belongs to $B$. As a consequence, there exists a unique admissible cut $\boldsymbol{v}$ such that the vertices of $\mathcal{F}$ labelled by those subscripts $k$ such that $c_{i_{k} j_{k}}$ belongs to $B$ is Lea $\mathcal{F}_{\boldsymbol{F}}$ and the vertices of $\mathcal{F}$ indexed by those subscripts $k$ such that $c_{i_{k} j_{k}}$ belongs to $A$ is $\operatorname{Roo}_{\boldsymbol{v}} \mathscr{F}$. Moreover, $w$ is a word appearing in $S^{\mathrm{Roo}_{v} \mathscr{F}}(A) \otimes S^{\mathrm{Lea}_{v} \mathscr{F}}(B)$. Conversely, any word appearing in $S^{\mathrm{Roov}_{\boldsymbol{v}} \mathscr{F}}(A) \otimes S^{\mathrm{Lea}_{\boldsymbol{v}} \mathscr{F}}(B)$ appears in $S^{\mathscr{F}}(A \oplus B)$. Thus,

$$
S^{\mathcal{F}}(A \oplus B)=\sum_{\boldsymbol{v} \models \mathscr{F}} S^{\mathrm{Roo}_{\boldsymbol{v}} \mathcal{F}}(A) \otimes S^{\mathrm{Lea}_{\boldsymbol{v}} \mathcal{F}}(B)=S^{\Delta(\mathcal{F})} .
$$

Example. Let $\mathcal{F}={ }^{2} \dot{\vartheta}_{1}^{3}$. Then

$$
S^{\mathcal{F}}=\sum_{\substack{w_{1}<w_{1}, w_{2}, w_{3} \\ w_{3} \prec w_{4}}} w_{1} w_{2} w_{3} w_{4}
$$

so that

$$
S^{\mathcal{F}}(A)=\sum_{\substack{i_{1}<i_{2} \\ i_{1}<i_{3}<i_{4}}} a_{i_{1} i_{1}} a_{i_{1} i_{2}} a_{i_{1} i_{3}} a_{i_{3} i_{4}}
$$

and

$$
\begin{aligned}
& S^{\mathcal{F}}(A \oplus B)=\sum a_{i_{1} i_{1}} a_{i_{1} i_{2}} a_{i_{1} i_{3}} a_{i_{3} i_{4}}+\sum a_{i_{1} i_{1}} b_{j_{2} j_{2}} a_{i_{1} i_{3}} a_{i_{3} i_{4}} \\
& +\sum a_{i_{1} i_{1}} a_{i_{1} i_{2}} a_{i_{1} i_{3}} b_{j_{4} j_{4}}+\sum \sum a_{i_{1} i_{1}} b_{j_{2} j_{2}} a_{i_{1} i_{3}} b_{j_{4} j_{4}} \\
& +\sum a_{i_{1} i_{1}} a_{i_{1} i_{2}} b_{j_{3} j_{3}} b_{j_{3} j_{4}}+\sum \sum a_{i_{1} i_{1}} b_{j_{2} j_{2}} b_{j_{3} j_{3}} b_{j_{3} j_{4}} \\
& +\sum b_{j_{1} j_{1}} b_{j_{1} j_{2}} b_{j_{1} j_{3}} b_{j_{3} j_{4}} \\
& =S^{\mathcal{F}}(A)+S^{:_{1}^{\bullet_{1}}}(A) S^{\bullet_{1}}(B)+S^{2} \ddot{\vartheta}_{1}^{3}(A) S^{\bullet_{1}}(B)+S^{\dot{\bullet}_{1}^{2}}(A) S^{\bullet_{1} \bullet_{2}}(B) \\
& +S^{\bullet_{1}^{2}}(A) S^{\dot{\bullet}_{1}^{2}}(B)+S^{\bullet_{1}}(A) S^{\bullet_{1}}:_{2}^{3}(B)+S^{\mathcal{F}}(B) \text {. }
\end{aligned}
$$

We shall give a second realization of $\mathbf{H}_{o}$ in Section 5.2.

3.3. Epimorphism to WQSym. Let us recall the definition of WQSym, the Hopf algebra of ord Quasi-Symmetric functions (cf. [15], [24]). This algebra has many interpretations, e.g., as the Solomon-Tits descent algebra [28], [24], as a centralizer 
algebra for a kind of Schur-Weyl duality [23], and as an algebra of nonlinear difference operators [22].

The packed word $u=\operatorname{pack}(w)$ associated with a word $w \in A^{*}$ (over an ordered alphabet $A$ ) is obtained by the following process. If $b_{1}<b_{2}<\cdots<b_{r}$ are the letters occurring in $w$, then $u$ is the image of $w$ by the homomorphism $b_{i} \mapsto a_{i}$. A word $u$ is said to be packed if $\operatorname{pack}(u)=u$. The natural basis of WQSym, which lifts the quasi-monomial basis of QSym, is labelled by packed words. It is defined by

$$
\mathbf{M}_{u}=\sum_{\operatorname{pack}(w)=u} w
$$

In this basis, the product is given by

$$
\mathbf{M}_{u} \mathbf{M}_{v}=\sum_{\substack{w=u^{\prime} v^{\prime} \\ \operatorname{pack}\left(u^{\prime}\right)=u, \operatorname{pack}\left(v^{\prime}\right)=v}} \mathbf{M}_{v} .
$$

Let $\pi$ be the algebra morphism $a_{i j} \mapsto a_{j}$ from the free associative algebra on the $a_{i j}$ to the free associative algebra over single-indexed letters $a_{j}$.

Proposition 3.3. WQSym is a quotient Hopf algebra of $\mathbf{H}_{o}$ :

$$
\pi\left(\mathbf{H}_{o}\right)=\mathbf{W Q S y m} .
$$

Proof. Let $\mathscr{F}$ be an ordered forest with $n$ vertices. A packed word $m=a_{1} \ldots a_{n}$ is $\mathcal{F}$-admissible if $i \rightarrow j$ in $\mathcal{F}$ implies that $a_{j}<a_{i}$. Then

$$
\pi(\mathcal{F})=\sum_{m \mathcal{F} \text {-admissible }}\left(\sum_{\text {pack }(w)=m} w\right)=\sum_{m \mathcal{F} \text {-admissible }} \mathbf{M}_{m} .
$$

So $\pi(\mathcal{F}) \in$ WQSym.

Let us prove the surjectivity of $\pi$. We totally order packed words by the lexicographic order. For any packed word $w=a_{1} \ldots a_{n}$, let us construct an ordered forest $\mathcal{F}_{w}$ of degree $n$ such that the smallest packed word appearing in $\pi\left(\mathcal{F}_{w}\right)$ is $w$. We proceed by induction on $n$. If $n=1$, then $w=(1)$, and we take $\mathscr{F}_{(1)}=\cdot_{1}$. Let us assume the result for any packed word with $n-1$ letters. We separate the construction of $\mathscr{F}_{w}$ into three cases.

(1) $1=a_{1}=a_{2} \leq a_{3} \cdots \leq a_{n}$. We then take $\mathcal{F}_{w}={ }_{{ }_{1}} \mathcal{F}_{a_{2} \ldots a_{n}}$.

(2) $1=a_{1}<a_{2} \leq a_{3} \leq \cdots \leq a_{n}$. Then $a_{2}=2$. Let $a_{2}^{\prime} \ldots a_{n}^{\prime}=\operatorname{pack}\left(a_{2} \ldots a_{n}\right)$, that is to say $a_{i}^{\prime}=a_{i}-1$ for all $i$. We then take $\widetilde{\mathcal{F}}_{w}=B^{+}\left(\mathcal{F}_{a_{2}^{\prime} \ldots a_{n}^{\prime}}\right)$, that is to say the ordered tree obtained by adding a root to $\mathcal{F}_{a_{2}^{\prime}}^{\prime} \ldots a_{n}^{\prime}$, this root being the smallest element.

(3) The letters $a_{1}, \ldots, a_{n}$ are not in order. There exists $\sigma \in \mathbb{S}_{n}$ such that $a_{\sigma^{-1}(1)} \ldots a_{\sigma^{-1}(n)}$ is nondecreasing. We then take $\mathcal{F}_{w}=\sigma \cdot \mathcal{F}_{\sigma^{-1}(1)} \ldots a_{\sigma^{-1}(n)}$, where $\sigma$ acts by changing the order of the vertices of $\mathcal{F}_{a_{\sigma^{-1}(1)} \ldots a_{\sigma^{-1}(n)}}$. 
It is not difficult to show that these $\mathscr{F}_{w}$ give the result. So $\pi$ is surjective.

For example,

$$
\begin{aligned}
\pi\left(\bullet_{1}\right) & =\mathbf{M}_{(1)}, \\
\pi\left(\bullet_{1} \bullet_{2}\right) & =\mathbf{M}_{(11)}+\mathbf{M}_{(12)}+\mathbf{M}_{(21)}, \\
\pi\left(:_{1}^{2}\right) & =\mathbf{M}_{(12)}, \\
\pi\left(:_{2}^{1}\right) & =\mathbf{M}_{(21)}, \\
\pi\left({ }^{2} \grave{\vartheta}_{1}^{3}\right) & =\mathbf{M}_{(122)}+\mathbf{M}_{(132)}+\mathbf{M}_{(123)}, \\
\pi\left({ }^{1} \grave{\vartheta}_{3}^{2}\right) & =\mathbf{M}_{(221)}+\mathbf{M}_{(321)}+\mathbf{M}_{(231)} .
\end{aligned}
$$

3.4. Embedding of the noncommutative Connes-Kreimer algebra. Let $\overline{\mathcal{F}}$ be a plane forest. It can be seen as an ordered forest by totally ordering the vertices of $\mathcal{F}$ "up-left", that is, by performing a left depth-first traversal of the forest and numbering each vertex on the first encounter. For example,

$$
\dot{v} \dot{v} \mapsto{ }^{2} \dot{V}_{1}^{4}{ }^{4}{ }^{7} \dot{\gamma}_{5}^{8} .
$$

Proposition 3.4. The map $\overline{\mathcal{F}} \mapsto S^{\mathscr{F}}$ is a Hopf embedding of the noncommutative Connes-Kreimer algebra $\mathbf{H}_{\mathrm{NCK}}$ into $\mathbf{H}_{o}$.

Proof. This is clearly compatible with the product since shifted concatenation preserves the planar structure, and with the coproduct which is given on both sides by admissible cuts, the labeling having been chosen such that in $\mathbf{H}_{o}$ the coproduct of the image of a plane forest contains only terms corresponding to plane forests.

Thus, we have also a polynomial realization of $\mathbf{H}_{\mathrm{NCK}}$. For example,

$$
: \ddot{\vee} \mapsto S^{:_{1}^{2_{1}^{4}} \dot{\vartheta}_{3}^{6}}=\sum_{\substack{i_{1}<i_{2} \\ i_{3}<i_{4} \\ i_{3}<i_{5}<i_{6}}} a_{i_{1} i_{1}} a_{i_{1} i_{2}} a_{i_{3} i_{3}} a_{i_{3} i_{4}} a_{i_{3} i_{5}} a_{i_{5} i_{6}} .
$$

\subsection{Embedding of $\mathbf{H}_{\mathrm{NCK}}$ into WQSym}

Theorem 3.5. Let $\pi: \mathbf{H}_{o} \rightarrow \mathbf{W Q S y m}$ be the projection induced by $a_{i j} \mapsto a_{j}$ in the second realization. Then the restriction of $\pi$ to $\mathbf{H}_{\mathrm{NCK}}$ is injective.

Proof. Let $B_{+}$denote as usual the operation consisting of connecting the trees of a (plane) forest to a common root labeled 1 and shifting by one the labels of the trees. Define a (linear) endomorphism $b$ of WQSym by

$$
b\left(\mathbf{M}_{u}\right)=M_{1 \cdot u[1]},
$$


where is the concatenation and $u[1]$ means shifting by 1 the letters of $u$, e.g., $b\left(\mathbf{M}_{2131}\right)=\mathbf{M}_{13242}$.

We then have

$$
\pi \circ B_{+}=b \circ \pi .
$$

An ordered forest $\mathcal{F}_{\text {can }}$ be regarded as a poset $P_{\mathcal{F}}$ (the roots being minimal elements). Identifying a map $f$ from $P_{\mathscr{F}}$ to some $[m]$ with the word $w_{f}=$ $f(1) f(2) \ldots f(n)$, we have

$$
\pi\left(S^{\mathcal{F}}\right)=\sum_{f \in \mathcal{S}\left(P_{\mathscr{F}}\right)} \mathbf{M}_{w_{f}},
$$

where $S\left(P_{\mathscr{F}}\right)$ is the set of increasing surjections from $P_{\mathscr{F}}$ to some $[m]$. Now if we restrict to the $\mathcal{F}$ that are the canonical labelings of plane forests, the lexicographically minimal increasing surjection words $w_{f}$ are all distinct and hence allow to reconstruct $\mathcal{F}$. So the images of the plane forests are linearly independent.

3.6. The noncommutative Faà di Bruno algebra. Recall that the Faà di Bruno algebra is the Hopf algebra of polynomial functions on the group of formal diffeomorphisms of the real line tangent to the identity [5].

As an algebra, it can be identified with the algebra Sym of symmetric functions. The $n$-th coordinate function $f(t)=\sum f_{n} t^{n+1} \mapsto f_{n}$ of the Faà di Bruno algebra can be identified with the $n$-th complete symmetric homogeneous function $h_{n}(X)$, i.e., the sum of all monomials of degree $n$. In terms of generating series,

$$
\sigma_{t}:=\sum_{n} t^{n} h_{n}(X)=\prod_{i \geq 1}\left(1-t x_{i}\right)^{-1} .
$$

For a scalar $\alpha$, we define the notation $h_{n}(\alpha X)$ by

$$
\sigma_{t}(\alpha X)=\sum_{n} t^{n} h_{n}(\alpha X)=\sigma_{t}(X)^{\alpha} .
$$

With this identification, the coproduct of the Faà di Bruno acts on complete homogeneous functions $h_{n}$ by

or, equivalently,

$$
\Delta_{1} h_{n}=\sum_{k=0}^{n} h_{k}(X) h_{n-k}((k+1) Y)
$$

$$
\Delta \sigma_{1}=\sum_{n \geq 0} h_{n} \otimes \sigma_{1}^{n+1},
$$

The noncommutative version of [7], [1] can be identified with the algebra Sym of noncommutative symmetric functions [11], [26], endowed with the coproduct

$$
\Delta_{1} S_{n}=\sum_{k=0}^{n} S_{k}(A) S_{n-k}((k+1) B)
$$


or again

$$
\Delta \sigma_{1}=\sum_{n \geq 0} S_{n} \otimes \sigma_{1}^{n+1},
$$

where the $S_{n}(\alpha A)$ are defined by

$$
\sigma_{t}(A)=\sum_{n} t^{n} S_{n}(A)=\prod_{i \geq 1}\left(1-t a_{i}\right)^{-1} \quad \text { and } \quad \sigma_{t}(\alpha A)=\sigma_{t}(A)^{\alpha} .
$$

The Faà di Bruno algebra is known to be a Hopf subalgebra of the Connes-Kreimer algebra, and in the same way its noncommutative version can be embedded in $\mathbf{H}_{\mathrm{NCK}}$ [7]. Let

$$
U=\sum_{F} F=\frac{1}{1-V} \quad \text { and } \quad V=\sum_{T} T=B_{+}(U)
$$

be the sum of all plane forests and the sum of all plane trees in $\mathbf{H}_{\mathrm{NCK}}$. It is shown in [7] that the square $Z=U^{2}$ of $U$ has the same coproduct as $\sigma_{1}$ :

$$
\Delta_{\mathrm{NCK}} Z=\sum_{n \geq 0} Z_{n} \otimes Z^{n+1} .
$$

Thus, composing the map $S_{n} \mapsto Z_{n}$ with the embedding of $\mathbf{H}_{\mathrm{NCK}}$ into WQSym, we obtain an embedding of the noncommutative Faà di Bruno algebra.

3.7. Epimorphism to the original Connes-Kreimer algebra. If, in the above realization of $\mathbf{H}_{\mathrm{NCK}}$, we map $a_{i j} \mapsto x_{i j}$ where the $x_{i j}$ are commuting indeterminates, we then obtain a commutative Hopf algebra which turns out to be the original ConnesKreimer algebra. We can even do this at the level of $\mathbf{H}_{o}$. With both realizations, $S^{\mathcal{F}}$ and $S^{\mathscr{E}}$ have the same image if and only if the underlying unordered forests are the same. Thus the image of $\mathbf{H}_{o}$ is also the Connes-Kreimer algebra.

Proposition 3.6. The map $a_{i j} \mapsto x_{i j}$ provides a polynomial realization of the Connes-Kreimer algebra.

Proof. The fact that $S^{\mathcal{F}}(X)$ depends only on the underlying forests is clear from the definition. Compatibility with the product and coproduct is also immediate. The only point which has to be checked is that the map is surjective. This follows from the same argument as in the proof of Theorem 3.2.

The commutative images of the polynomials $S^{\mathscr{F}}$ are special cases of polynomials known in numerical analysis, as well as their coproduct formula (see, e.g., [14]). More precisely, the specialization of these polynomials $S^{\mathcal{F}}$ to the coefficients of a finite matrix gives the polynomials associated with each tree by a Runge-Kutta method (here, with a triangular matrix). The direct construction of the coproduct in terms of the $\prec$-alphabets presented here is new. 
3.8. Analog of the Schur basis. The basis $S^{\mathscr{F}}$ is multiplicative in the sense that the product of two basis elements is again a basis element. In general, combinatorial Hopf algebras admit several interesting bases, and such multiplicative bases are generally obtained by summing some other combinatorial basis along intervals on some order. This is the case here.

There is a natural order on ordered forests with a given number $n$ of vertices, whose cover relation is $\mathscr{F}^{\prime}<\mathscr{F}^{\prime}$ if and only if $\mathscr{F}^{\prime}$ is obtained from $\mathscr{F}$ by deleting exactly one edge. In other words, considering the egdes of $\mathscr{F}$ and $\mathscr{F}^{\prime}$ as elements of $\{1, \ldots, n\}^{2}, \mathscr{F} \leq \mathscr{F}^{\prime}$ if and only if the set of edges of $\mathscr{F}^{\prime}$ is included in the set of edges of $\mathscr{F}$.

Let us set

$$
R_{\mathscr{F}}=\sum_{\mathscr{E} \leq \mathcal{F}}(-1)^{|E(\mathcal{F})|-|E(\mathscr{E})|} S^{\mathscr{E}} .
$$

For example,

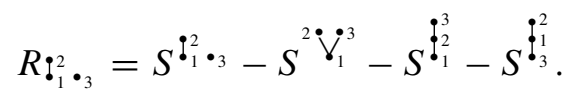

Let $\mathcal{F}$ be a forest with $k$ vertices and let $I \subseteq\{1, \ldots, k\}$. The restriction $\mathcal{F}_{\mid I}$ is the subforest of $\mathcal{F}$ obtained by taking all the vertices of $\mathscr{F}$ which are in $I$ and all the edges between these vertices. As $I$ is totally ordered (as a part of $\{1, \ldots, k\}$ ), $\widetilde{F}_{\mid I}$ is an ordered forest. Hence

Theorem 3.7. Let $\mathcal{F}^{\prime}$ and $\mathcal{F}^{\prime \prime}$ be two ordered forests, with $k^{\prime}$ and $k^{\prime \prime}$ vertices, respectively. Then

$$
R_{\mathcal{F}^{\prime}} R_{\mathscr{F}^{\prime \prime}}=\sum R_{\mathscr{F}},
$$

where the sum is over all ordered forests $\mathscr{F}$ with $k^{\prime}+k^{\prime \prime}$ vertices such that $\mathscr{F}_{\mid} \mid\left\{1, \ldots, k^{\prime}\right\}=$ $\mathscr{F}^{\prime}$ and $\mathscr{F}_{\mid\left\{k^{\prime}+1, \ldots, k^{\prime}+k^{\prime \prime}\right\}}=\mathscr{F}^{\prime \prime}$.

Proof. Let us define another product $\star$ on $\mathbf{H}_{o}$, given by the formula we want to prove. Let us then compute $S^{\mathcal{F}^{\prime} \star \mathcal{F}^{\prime \prime}}$ for any ordered forests $\mathscr{F}^{\prime}$ and $\mathscr{F}^{\prime \prime}$. By a Möbius inversion, for any ordered forest $\mathcal{G}$,

$$
S^{\mathscr{E}}=\sum_{\mathscr{E}^{\prime} \leq \mathscr{E}} R_{\mathscr{E}^{\prime}},
$$

so that

$$
S^{\mathcal{F}^{\prime} \star \mathcal{F}^{\prime \prime}}=\sum_{\mathscr{E}^{\prime} \leq \mathcal{F}^{\prime}, \mathscr{S}^{\prime \prime} \leq \mathcal{F}^{\prime \prime}} R_{\mathscr{G}^{\prime} \star R_{\mathcal{E}^{\prime \prime}}}=\sum R_{\mathscr{g}},
$$

where the sum is over all ordered forests $\mathscr{\mathscr { C }}$ with $k^{\prime}+k^{\prime \prime}$ vertices such that $\mathscr{P}_{\mid\left\{1, \ldots, k^{\prime}\right\}} \leq$ $\mathscr{F}^{\prime}$ and $\mathscr{E}_{\mid\left\{k^{\prime}+1, \ldots, k^{\prime}+k^{\prime \prime}\right\}} \leq \mathscr{F}^{\prime \prime}$. Such an ordered forest $\mathcal{H}$ is obtained, first by adding edges between vertices of $\mathscr{F}^{\prime}$ and $\mathcal{F}^{\prime \prime}$, then edges between vertices of the two ordered forests. Equivalently, it can be obtained by adding edges between vertices of $\mathscr{F}^{\prime} \mathscr{F}^{\prime \prime}$ so that

$$
S^{\mathscr{F}^{\prime} \star \mathscr{F}^{\prime \prime}}=\sum_{\mathscr{E} \leq \mathcal{F}^{\prime} \mathscr{F}^{\prime \prime}} R R_{\mathscr{g}}=S^{\mathcal{F}^{\prime} \mathcal{F}^{\prime \prime}}=S^{\mathcal{F}^{\prime}} S^{\mathscr{F}^{\prime \prime}} .
$$

So $\star$ is the product of $\mathbf{H}_{o}$. 
For example,

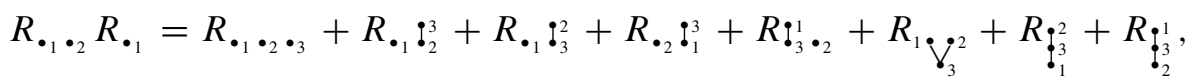

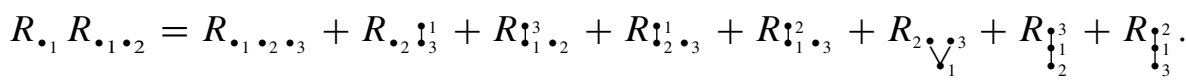

3.9. A Schur basis for the Connes-Kreimer algebra. Let $\mathcal{F}$ and $\mathcal{F}^{\prime}$ be two ordered forests with the same underlying rooted forest. There exists a permutation $\sigma$ such that the ordered forest $\mathcal{F}^{\sigma}$ obtained from $\mathcal{F}$ by permuting the indices by $\sigma$ is equal to $\mathcal{F}^{\prime}$. Then $\mathcal{E}^{\sigma} \leq \mathcal{F}^{\prime}$ for any ordered forest $\mathcal{E} \leq \mathcal{F}$ since $\mathcal{E}$ is obtained from $\mathscr{F}$ by adding some edges. As a consequence, the commutative images of $R^{\mathscr{F}}$ and $R^{\mathscr{F}^{\prime}}$ are equal. For any rooted forest $\overline{\mathcal{F}}$, we then denote by $R^{\overline{\mathcal{F}}}$ the image of $R^{\mathscr{F}}$ in the Connes-Kreimer algebra, where $\mathscr{F}$ is any ordered forest with underlying rooted forest $\overline{\mathcal{F}}$; this does not depend on the choice of $\mathcal{F}$. These elements form a new basis of the Connes-Kreimer Hopf algebra.

Examples. In the Connes-Kreimer Hopf algebra,

$$
\begin{aligned}
& R^{\bullet}=\cdot, \quad R^{\natural}=!, \quad R^{\bullet}=\ldots-2 !,
\end{aligned}
$$

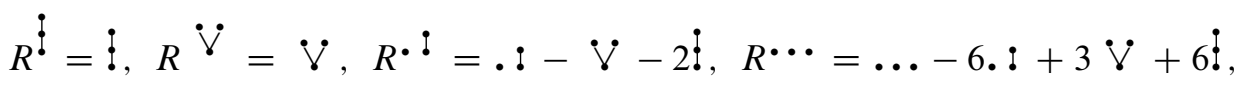

$$
\begin{aligned}
& R^{\vdots}=\vdots \\
& R^{\dddot{y}}=\ddot{Y} \\
& R \stackrel{\dot{v}}{\dot{v}} \dot{v} \\
& R{ }^{\dddot{V}}=\ddot{\vee} \\
& R^{:}:=: !-2 \dot{\vee}-2 \dot{\text {, }} \\
& R^{\ddagger}=\vdots-\grave{\gamma}-\grave{\gtrless}-2 \vdots \text {, } \\
& R \ddot{V} \cdot=\ddot{V} \cdot-\ddot{V}-2 \ddot{V}-\ddot{Y},
\end{aligned}
$$

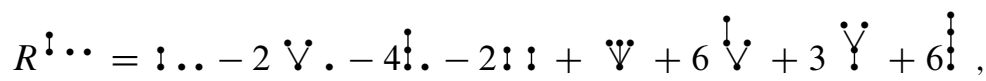

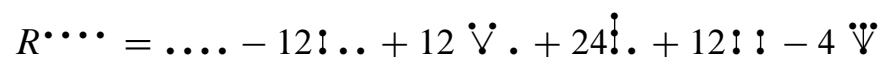

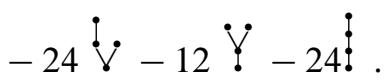




\section{The cocommutative Hopf algebra on permutations}

Besides the self dual Hopf algebra structure (known as FQSym or as the MalvenutoReutenauer algebra [21]) on the linear span of all permutations, there is another one which is cocommutative and noncommutative. It was first described by Grossman and Larson [13] in terms of heap ordered trees. Several other (non-obviously equivalent) constructions can be found in [18].

The starting point of [18] is a commutative algebra, denoted by SQSym, spanned by the polynomials

$$
M_{\sigma}=\sum_{i_{1}<\cdots<i_{n}} x_{i_{1} i_{\sigma(1)}} \ldots x_{i_{n} i_{\sigma(n)}}
$$

in commuting indeterminates $x_{i j}$ satisfying $x_{i j} x_{i k}=x_{i k} x_{j k}=0$. The dual Hopf algebra $\mathbf{S} \mathbf{S y m}$ is free over the set of connected permutations, and the dual basis $\mathbf{S}^{\sigma}$ of $M_{\sigma}$ satisfies

$$
\mathbf{S}^{\sigma} \mathbf{S}^{\tau}=\mathbf{S}^{\sigma \bullet \tau}
$$

where $\bullet$ denotes the shifted concatenation [18], defined by

$$
\left(a_{1}, \ldots, a_{m}\right) \bullet\left(b_{1}, \ldots, b_{n}\right)=\left(a_{1}, \ldots, a_{m}, b_{1}+m, \ldots, b_{n}+m\right) .
$$

Theorem 4.1. Let $A=\left\{a_{i j} \mid i, j \geq 1\right\}$ endowed with the relation $a_{i j} \prec a_{k l}$ if and only if $j=k$. Then the polynomials

$$
\mathbf{S}^{\sigma}(A):=\sum_{i_{1}, \ldots, i_{n} \geq 1} a_{i_{\sigma(1)} i_{1}} \ldots a_{i_{\sigma(n)} i_{n}}
$$

satisfy (1). Moreover, if $(B, \prec)$ is another alphabet, their sum $A \oplus B$ is defined as their disjoint union endowed with the $\prec$-relation restricting to the original ones of $A$ and $B$ and such that

$$
a_{i j} \prec b_{k l} \text { for all } i, j, k, l \text {. }
$$

Then these polynomials span a Hopf algebra isomorphic to $\mathbf{S S y m}$ for the coproduct $\Delta F(A)=F(A \oplus B)$.

Proof. The independence of the $\mathbf{S}^{\sigma}$ is proved in the same way as for Theorem 3.2: indeed, in any $\mathbf{S}^{\sigma}(A)$ appears a word such that all subscripts $i_{k}$ are different and such a word allows one to rebuild $\sigma$. Moreover, the $\mathbf{S}^{\sigma}$ defined by (2) satisfy (1). For the coproduct, observe that $\mathbf{S}^{\sigma}(A)$ can alternatively be characterized as the sum of all $\sigma$-compatible words, defined by the condition

$$
w=a_{k_{1} l_{1}} \ldots a_{k_{n} l_{n}} \text { is } \sigma \text {-compatible if and only if } i=\sigma(j) \Longrightarrow a_{k_{i} l_{i}} \prec a_{k_{j} l_{j}} \text {. }
$$

Hence, $\mathbf{S}^{\sigma}(A \oplus B)$ is well defined and obtained from $\mathbf{S}^{\sigma}(A)$ by splitting the set of cycles of $\sigma$ into two parts in all possible ways and replacing $a$ 's by $b$ 's into one of the parts. This is exactly the coproduct of the basis $\mathbf{S}^{\sigma}$ of $\mathbf{S S y m}$ as described in [18]. 
Example. Let us consider $\sigma=(24513)$. Then

$$
\mathbf{S}^{\sigma}(A)=\sum_{i_{1}, i_{2}, i_{3}, i_{4} \geq 1} a_{i_{2} i_{1}} a_{i_{4} i_{2}} a_{i_{5} i_{3}} a_{i_{1} i_{4}} a_{i_{3} i_{5}},
$$

so that

$$
\mathbf{S}^{\sigma}=\sum_{\substack{w_{1} \prec w_{4}<w_{2} \prec w_{1} \\ w_{3} \prec w_{5} \prec w_{3}}} w_{1} w_{2} w_{3} w_{4} w_{5}
$$

Hence,

$$
\begin{aligned}
\mathbf{S}^{\sigma}(A \oplus B)= & \sum a_{i_{2} i_{1}} a_{i_{4} i_{2}} a_{i_{5} i_{3}} a_{i_{1} i_{4}} a_{i_{3} i_{5}}+\sum a_{i_{2} i_{1}} a_{i_{4} i_{2}} b_{i_{5} i_{3}} a_{i_{1} i_{4}} b_{i_{3} i_{5}} \\
& +\sum b_{i_{2} i_{1}} b_{i_{4} i_{2}} a_{i_{5} i_{3}} b_{i_{1} i_{4}} a_{i_{3} i_{5}}+\sum b_{i_{2} i_{1}} b_{i_{4} i_{2}} b_{i_{5} i_{3}} b_{i_{1} i_{4}} b_{i_{3} i_{5}} \\
= & \mathbf{S}^{\sigma}(A)+\mathbf{S}^{(231)}(A) \mathbf{S}^{(12)}(B)+\mathbf{S}^{(12)}(A) \mathbf{S}^{(231)}(B)+\mathbf{S}^{\sigma}(B) .
\end{aligned}
$$

\section{A Hopf algebra of endofunctions}

5.1. Construction. The commutative Hopf algebra of permutations of [18] is actually a subalgebra and a quotient of a commutative algebra based on endofunctions, i.e., maps from $\{1,2, \ldots, n\}$ to itself. There is a similar construction here.

Let $A=\left\{a_{i j} \mid i \neq j, i, j \geq 1\right\}$, endowed with the relation $a_{i j} \prec a_{k l}$ if and only if $j=k$. For a function $f:[n] \rightarrow[n]$, let us say that a word $w=w_{1} \ldots w_{n}$ is $f$-compatible if and only if $i \neq j$ and $i=f(j)$ imply that $w_{i} \prec w_{j}$. Define

$$
\mathbf{S}^{f}(A):=\sum_{w f \text {-compatible }} w .
$$

For example, representing a function as the list of its images, if $f=(24352)$, one has

$$
\mathbf{S}^{f}=\sum_{\substack{w_{2}<w_{1} \\ w_{2} \prec w_{5} \prec w_{4} \prec w_{2}}} w_{1} w_{2} w_{3} w_{4} w_{5}=\sum_{i \neq k, j, n, k \neq n, l \neq m} a_{i j} a_{k i} a_{l m} a_{n k} a_{i n} .
$$

Note that, as before, these elements are linearly independent: any monomial in $\mathbf{S}^{f}$ with as many different subscripts as possible allows one to reconstruct the relations $w_{i}<w_{j}$ and hence the images of $f$ (fixed points being the missing ones).

Theorem 5.1. The $\mathbf{S}^{f}$ span a subalgebra of $\mathbb{K}\langle A\rangle$, with

$$
\mathbf{S}^{f} \mathbf{S}^{g}=\mathbf{S}^{f \bullet g},
$$

where, again, $\bullet$ denotes the shifted concatenation. Moreover, if $(B, \prec)$ is another alphabet, their sum $A \oplus B$ is defined as their disjoint union endowed with the $\prec-$ relation restricting to the original ones of $A$ and $B$ and such that

$$
a_{i j} \prec b_{k l} \quad \text { for all } i \neq j, k \neq l \text {. }
$$


Then these polynomials span a (non-cocommutative) Hopf algebra for the coproduct $\Delta \mathbf{S}^{f}=\mathbf{S}^{f}(A \oplus B)$.

Proof. Similar to the proof of Theorem 4.1.

Let us give a description of the coproduct. Let $f:[n] \rightarrow[n]$ and let $I \subseteq[n]$. Let $f^{I}: I \rightarrow I$ be the map satisfying $f^{I}(x)=f(x)$ if $f(x) \in I$ and $f^{I}(x)=x$ otherwise. If $I$ has cardinality $k$, there exists a unique increasing bijection $\tau_{I}: I \rightarrow$ $[k]$. Then $\operatorname{Std}\left(f^{I}\right):=\tau_{I} \circ f^{I} \circ \tau_{I}^{-1}$. We shall say that $I$ is an ideal of $f$ and write $I \models f$ if $f^{-1}(I) \subseteq I$.

One then sees that

$$
\Delta\left(S^{f}\right)=\sum_{I \models f} S^{\operatorname{Std}\left(f^{[n] \backslash I}\right)} \otimes S^{\operatorname{Std}\left(f^{I}\right)} .
$$

Example. Let us consider $f=(23234)$. Then

$$
S^{f}=\sum_{\substack{w_{2}<w_{1}, w_{3} \\ w_{3}<w_{2}, w_{4} \\ w_{4}<w_{5}}} w_{1} w_{2} w_{3} w_{4} w_{5}
$$

so that

$$
S^{f}(A)=\sum_{\substack{j \neq i, k \\ l \neq k, m}} a_{j i} a_{k j} a_{j k} a_{k l} a_{l m}
$$

and

$$
\begin{aligned}
S^{f}(A \oplus B)= & \sum a_{j i} a_{k j} a_{j k} a_{k l} a_{l m}+\sum b_{q p} a_{k j} a_{j k} a_{k l} a_{l m}+\sum a_{j i} a_{k j} a_{j k} a_{k l} b_{q p} \\
& +\sum b_{q p} a_{k j} a_{j k} a_{k l} b_{r s}+\sum a_{j i} a_{k j} a_{j k} b_{p q} b_{q r} \\
& +\sum b_{q p} a_{k j} a_{j k} b_{r s} b_{s t}+\sum b_{q p} b_{r q} b_{q r} b_{r s} b_{s t} \\
= & S^{f}(A)+S^{(2123)}(A) S^{(1)}(B)+S^{(2323)}(A) S^{(1)}(B) \\
& +S^{(212)}(A) S^{(12)}(B)+S^{(232)}(A) S^{(11)}(B) \\
& +S^{(21)}(A) S^{(122)}(B)+S^{(f)}(B) .
\end{aligned}
$$

Hence,

$$
\begin{aligned}
\Delta\left(S^{f}\right)= & S^{f} \otimes 1+S^{(2123)} \otimes S^{(1)}+S^{(2323)} \otimes S^{(1)}+S^{(212)} \otimes S^{(12)} \\
& +S^{(232)} \otimes S^{(11)}+S^{(21)} \otimes S^{(122)}+1 \otimes S^{(f)} .
\end{aligned}
$$

Note that the ideals of $f$ are $\emptyset,\{1\},\{5\},\{1,5\},\{4,5\},\{1,4,5\}$, and $\{1,2,3,4,5\}$.

We shall give a graphical representation of endofunctions. If $f:[n] \rightarrow[n]$, the vertices of the graph associated with $f$ are the elements of $[n]$, and there is an edge 
from $i$ to $j$ if and only if $f(i)=j$ for all $i \neq j$. For example, the graph associated with (23234) is

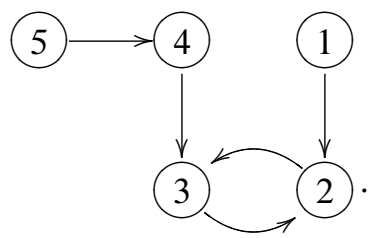

The ideals of $f$ are then given by admissible cuts of the graph (note that the edges in the cycles cannot be cut).

We shall denote this Hopf algebra of endofunctions by EFSym.

5.2. Hopf subalgebras and quotients. algebra of endofunctions, having dimension $n^{n}$ in degree $n$, is large enough to admit as subalgebras or quotients many combinatorial Hopf algebras, old and new. We shall not attempt to be exhaustive, and restrict ourselves to the description of new polynomial realizations of the previous ones, and to a short list of new algebras, which will be investigated elsewhere.

5.2.1. Permutations. The $\mathbf{S}^{\sigma}$, where $\sigma$ runs over permutations, span a Hopf subalgebra of EFSym isomorphic to SSym. Indeed, if $f$ and $g$ are permutations, then $f \bullet g$ is also a permutation; if $f$ is a permutation, then its ideals are the disjoint unions of cycles of $f$, so one recovers the Hopf algebra structure of SSym described in [18]. Note that the two realizations are different: in the realization of Section 4, $S^{(1)}=\sum_{i \geq 1} a_{i i}$ and $S^{(12)}=\sum_{i, j \geq 1} a_{i j} a_{i j}$; in the realization as endofunctions, $S^{(1)}=\sum_{i, j \geq 1} a_{i j}$ and $S^{(12)}=\sum_{i, j \geq 1, i \neq j} a_{i j} a_{j i}$.

5.2.2. Ordered forests. The $\mathbf{S}^{\phi}$, where $\phi$ runs over acyclic functions, span a Hopf subalgebra of EFSym isomorphic to our first algebra of labelled forests, hence to PQSym*. Indeed, if $\mathscr{F}$ is a labelled forest, we can define an acyclic function $f_{\mathscr{F}}$ in the following way: if there is an edge from $i$ to $j$ in $\mathcal{F}$, then $f(j)=i$. If $i$ is a root of $\mathcal{F}$, then $f_{\mathscr{F}}(i)=i$. For example,

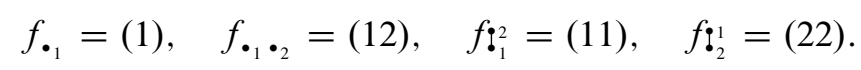

In other words, $f_{\mathscr{F}}$ is the endofunction whose graph is $\mathcal{F}$, the orientation being implicitly from top to bottom. Now, $f_{\mathscr{F}, \mathscr{E}}=f_{\mathscr{F}} \bullet f_{\mathscr{E}}$. Moreover, the ideals of $f_{\mathscr{F}}$ are the set of the indices $I$ such that the vertices of $\mathscr{F}$ indexed by $I$ are a Lea $\mathscr{F}$, where $v$ runs over the set of admissible cuts of $\mathcal{F}$. So

$$
\mathbf{H}_{o} \rightarrow \mathbf{E F S y m}, \quad S^{\mathcal{F}} \mapsto S^{f_{\mathcal{F}}},
$$

is an injective map of graded Hopf algebras. This gives a second realization of $\mathbf{H}_{o}$. These two realizations do not coincide; for example, if $\mathscr{F}=:_{2}^{1}$, then $S^{\mathscr{F}}=$ $\sum_{i \geq j \geq 1} a_{j i} a_{j j}$ and $S^{f_{F F}}=\sum_{j \neq i, k} a_{j i} a_{k j}$. 
5.2.3. Plane forests. We have seen that the noncommutative Connes-Kreimer algebra is a Hopf subalgebra of $\mathbf{H}_{o}$. Moreover, for any ordered forest $\mathcal{F}$, the acyclic function $f_{\mathscr{F}}$ is a nondecreasing parking function if and only if $\mathscr{F}$ is a plane forest. So the restriction of the embedding $S^{\mathscr{F}} \mapsto S^{f_{\mathscr{F}}}$ is an isomorphism from the noncommutative Connes-Kreimer Hopf algebra to the subspace spanned by the $\mathbf{S}^{\boldsymbol{\pi}}$, where $\pi$ runs over nondecreasing parking functions, which is then a Hopf subalgebra of EFSym. So this gives another realization of the noncommutative Connes-Kreimer algebra. These realizations are indeed different: for the realization of Section 3.2,

$$
S^{:_{1}^{2}}=\sum_{1 \leq i \leq j} a_{i i} a_{i j}
$$

whereas for the realization as endofunctions,

$$
S^{:_{1}^{2}}=\sum_{1 \leq i, j, k} a_{i j} a_{j k}
$$

Let $I_{o}$ be the subspace generated by the $S^{f}$, where $f$ runs over the set of endofunctions which are not acyclic. It is clear that $I_{o}$ is an ideal of the Hopf algebra of endofunctions. Moreover, if $f$ is not acyclic, then it contains a cycle $C=i_{1} \mapsto i_{2} \mapsto \cdots \mapsto i_{k} \mapsto i_{1}$ of length $\geq 2$. Let $I$ be an ideal of $f$. If $C \cap I \neq \emptyset$, then by definition of the ideals, $C \subseteq I$, so either $\operatorname{Std}\left(f^{I}\right) \operatorname{or} \operatorname{Std}\left(f^{[n]-I}\right)$ is not acyclic: this implies that $I_{o}$ is a Hopf ideal of the Hopf algebra of endofunctions. So the quotient $\mathbf{H}_{o} / I_{o}$ is isomorphic to the Hopf subalgebra of acyclic endofunctions, hence to $\mathbf{H}_{o}$ and PQSym*.

5.2.4. Nondecreasing sets. The restriction to nondecreasing functions also gives rise to a Hopf algebra: if $f$ and $g$ are nondecreasing functions, then $f \bullet g$ is also nondecreasing, and for any ideal $I$ of $f, \operatorname{Std}\left(f^{I}\right)$ and $\operatorname{Std}\left(f^{[n]-I}\right)$ are also nondecreasing.

5.2.5. Burnside classes. The restriction to idempotent functions or, more generally, to Burnside classes $\left(f^{p}=f^{q}\right.$ ) gives, as in the commutative case, Hopf subalgebras: if $f^{p}=f^{q}$ and $g^{p}=g^{q}$, then $(f \bullet g)^{p}=(f \bullet g)^{q}$ and $\operatorname{Std}\left(f^{I}\right)^{p}=\operatorname{Std}\left(f^{I}\right)^{q}$ for any part $I$ of the domain of $f$. Graphically, this corresponds to endofunctions $f$ such that the graph of $f$ contains only cycles of length dividing $|p-q|$ and trees of height smaller than $|p-q|$. In particular, for the idempotent functions, this gives endofunctions whose graph is a corolla, that is to say a tree of height at most 1 .

5.2.6. Commutative images. The commutative images of the $\mathbf{S}^{\phi}\left(a_{i j} \mapsto x_{i j}\right)$ span a commutative Hopf algebra containing the algebra Sym of ordinary symmetric functions (as the image of the subalgebra isomorphic to SSym) and the Connes-Kreimer Hopf algebra of trees (as the image of the subalgebra isomorphic to $\mathbf{H}_{o}$ ). 
5.3. Analog of the Schur basis. We define a partial order on the set of endofunctions of $[n]$ for a fixed $n$, whose cover relation is $f<g$ if there exists an element $j$ of $[n]$ with $f(j) \neq j$ such that $g(k)=f(k)$ if $k \neq j$ and $g(j)=j$. For example, for $n=2$, the Hasse graph of this partial order is:

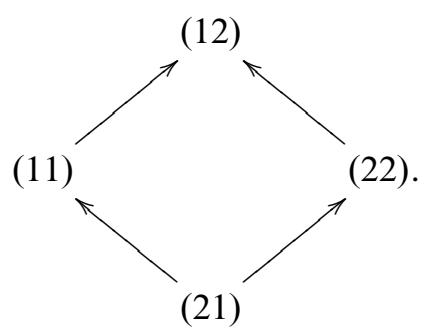

Note. Let $\mathcal{F}$ and $\mathcal{G}$ be two ordered forests. It is not difficult to show that $f_{\mathscr{F}} \leq f_{\mathscr{E}}$ if and only if $\mathscr{F} \leq \mathscr{E}$.

For any endofunction $f$, let us set

$$
R_{f}=\sum_{g \leq f}(-1)^{\operatorname{Fix}(f)-\operatorname{Fix}(g)} S^{g},
$$

where $\operatorname{Fix}(f)$ denotes the number of fixed points of $f$. By a Möbius inversion, for any endofunction $f$, we mean

$$
S^{f}=\sum_{g \leq f} R_{g}
$$

In analogy with Theorem 3.7 , one can show

Theorem 5.2. Let $f^{\prime}$ and $f^{\prime \prime}$ be two endofunctions of respectively $\left[n^{\prime}\right]$ and $\left[n^{\prime \prime}\right]$. Then

$$
R_{f^{\prime}} R_{f^{\prime \prime}}=\sum R_{f}
$$

where the sum is over all endofunctions $f$ of $\left[n^{\prime}+n^{\prime \prime}\right]$ such that $\operatorname{Std}\left(f^{\left[n^{\prime}\right]}\right)=f^{\prime}$ and $\operatorname{Std}\left(f^{\left[n^{\prime}+n^{\prime \prime}\right] \backslash\left[n^{\prime}\right]}\right)=f^{\prime \prime}$.

For example,

$$
\begin{aligned}
R_{(12)} R_{(1)}= & R_{(121)}+R_{(122)}+R_{(123)}+R_{(131)}+R_{(132)}+R_{(133)}, \\
& +R_{(321)}+R_{(322)}+R_{(323)}+R_{(331)}+R_{(332)}+R_{(333)}, \\
R_{(1)} R_{(12)}= & R_{(111)}+R_{(113)}+R_{(121)}+R_{(123)}+R_{(211)}+R_{(213)}, \\
& +R_{(221)}+R_{(223)}+R_{(311)}+R_{(313)}+R_{(321)}+R_{(323)} .
\end{aligned}
$$

Indeed, for $R_{(12)} R_{(1)}$, one gets all functions such that $f(1)$ is either 1 or 3 , and $f(2)$ is either 2 or 3 , the value $f(3)$ having no constraint at all. 
Let us consider the subspace $I_{o}^{\prime}$ generated by the $R_{f}$, where $f$ runs over the set of endofunctions $f$ which are not acyclic. If $f^{\prime}$ or $f^{\prime \prime}$ is not acyclic and if $R_{f}$ appears in $R_{f^{\prime}} R_{f^{\prime \prime}}$, then $f$ is not acyclic. So $I_{0}^{\prime}$ is an ideal. Let us denote by $\overline{R_{f}}$ the class of $R_{f}$ modulo $I_{0}^{\prime}$. Note that $\overline{R_{f}}$ is nonzero if and only if $f$ is acyclic, that is, there exists an ordered forest $\mathscr{F}$ such that $f=f_{\mathscr{F}}$. Moreover, if $\mathscr{F}$ is an ordered forest with $n$ vertices and $I \subseteq[n]$, then $\mathscr{F}_{\mid I}=\mathscr{E}$ if and only if $\operatorname{Std}\left(f_{\mathscr{F}}^{I}\right)=f_{\mathscr{E}}$. So the map $R_{\mathscr{F}} \mapsto \bar{R}_{f_{\mathscr{F}}}$ is an algebra isomorphism from the algebra $\mathbf{H}_{o}$ to the algebra of endofunctions quotiented by $I_{o}^{\prime}$.

Remark. The ideals $I_{o}$ and $I_{o}^{\prime}$ are different: in degree 2, $I_{o}$ is spanned by $S^{21}$, whereas $I_{0}^{\prime}$ is generated by $R_{21}=S^{(21)}-S^{(11)}-S^{(22)}+S^{(12)}$.

\section{Conclusion}

Polynomial realizations of combinatorial Hopf algebras are important for at least two reasons. First, a realization usually leads to important simplifications in the theory. Next, this may lead to the definition of new families of special functions, analogous to the Schur, Hall-Littlewood or Macdonald functions of the classical theory of symmetric functions. We have made a small step in this direction by introducing Schur-like bases in algebras of the Connes-Kreimer family and in those derived from the graphical representations of endofunctions. The main novelty in this paper is the idea that the $A+B$ trick for the coproduct can be implemented with $\prec$-relations on alphabets which are not order relations as in all the previously known examples.

Here is a short table summarizing the $\prec$-relations introduced in this paper.

\begin{tabular}{|c|c|c|c|c|}
\hline Algebra & Alphabet & Relation $\prec$ & Sum of alphabets & words \\
\hline $\mathbf{H}_{o}$ & $\begin{array}{c}a_{i j}, 1 \leq i \leq j \\
\text { noncommutative }\end{array}$ & $a_{i j} \prec a_{j k}$ & $a_{i j} \prec b_{k k}$ & $\begin{array}{c}l \rightarrow k \\
\Rightarrow w_{k} \prec w_{l}\end{array}$ \\
\hline $\mathbf{H}_{\mathrm{CK}}$ & $\begin{array}{c}x_{i j}, 1 \leq i \leq j \\
\text { commutative }\end{array}$ & $x_{i j} \prec x_{j k}$ & $x_{i j} \prec x_{k k}$ & $\begin{array}{c}l \rightarrow k \\
\text { SSym }\end{array}$ \\
$\begin{array}{c}a_{i j}, 1 \leq i, j, i \neq j \\
\text { noncommutative }\end{array}$ & $a_{i j} \prec a_{j k}$ & $a_{i j} \prec b_{k l}$ \\
\hline EFSym & $\begin{array}{c}a_{i j}, 1 \leq i, j, i \neq j \\
\text { noncommutative }\end{array}$ & $a_{i j} \prec a_{j k}$ & $a_{i j} \prec b_{k l}$ & $\begin{array}{c}l \rightarrow k \\
l\end{array}$ \\
\hline
\end{tabular}




\section{References}

[1] C. Brouder, A. Frabetti, and C. Krattenthaler, Non-commutative Hopf algebra of formal diffeomorphisms. Adv. Math. 200 (2006), 479-524. Zbl 1133.16025 MR 2200854

[2] F. Chapoton and M. Livernet, Pre-Lie algebras and the rooted trees operad. Internat. Math. Res. Notices (2001), 395-408. Zbl 1053.17001 MR 1827084

[3] A. Connes and D. Kreimer, Hopf algebras, renormalization and noncommutative geometry. Comm. Math. Phys. 199 (1998), 203-242. Zbl 0932.16038 MR 1660199

[4] G. Duchamp, F. Hivert, and J.-Y. Thibon, Noncommutative symmetric functions VI: Free quasi-symmetric functions and related algebras. Internat. J. Algebra Comput. 12 (2002), 671-717. Zbl 1027.05107 MR 1935570

[5] H. Figueroa and J. M. Gracia-Bondía, Combinatorial Hopf algebras in quantum field theory I. Rev. Math. Phys. 17 (2005), 881-976. Zbl 1090.16016 MR 2167639

[6] L. Foissy, Les algèbres de Hopf des arbres enracinés décorés, I. Bull. Sci. Math. 126 (2002), 193-239. Zbl 1013.16026 MR 1905177

[7] L. Foissy, Les algèbres de Hopf des arbres enracinés décorés, II. Bull. Sci. Math. 126 (2002), 249-288. Zbl 1013.16027 MR 1909461

[8] L. Foissy, Bidendriform bialgebras, trees, and free quasi-symmetric functions. J. Pure Appl. Algebra 209 (2007), 439-459. Zbl 1123.16030 MR 2293319

[9] L. Foissy, Ordered forests and parking functions. Internat. Math. Res. Notices 2012 (2012), 1603-1633. Zbl 1242.16031 MR 2913185

[10] L. Foissy and J. Unterberger, Ordered forests, permutations, and iterated integrals. Internat. Math. Res. Notices 2013 (2013), 846-885. Zbl MR 3024267

[11] I. M. Gelfand, D. Krob, A. Lascoux, B. Leclerc, V. S. Retakh, and J.-Y. Thibon, Noncommutative symmetric functions. Adv. Math. 112 (1995), 218-348. Zbl 0831.05063 MR 1327096

[12] R. Grossman and R. G. Larson, Hopf-algebraic structure of combinatorial objects and differential operators. Israel J. Math. 72 (1990), 109-117. Zbl 0780.16029 MR 1098983

[13] R. L. Grossman and R. G. Larson, Hopf algebras of heap ordered trees and permutations. Comm. Algebra 37 (2009), 453-459. Zbl 1161.16029 MR 2493713

[14] E. Hairer, C. Lubich, and G. Wanner, Geometric numerical integration. 2nd ed., Springer Ser. Comput. Math. 31, Springer-Verlag, Berlin 2006. Zbl 1094.65125 MR 2221614

[15] F. Hivert, Combinatoire des fonctions quasi-symétriques. Thèse de Doctorat, Université de Marne-La-Vallée, Marne-La-Vallée 1999.

[16] F. Hivert, J.-C. Novelli, and J.-Y. Thibon, Un analogue du monoïde plaxique pour les arbres binaires de recherche. C. R. Math. Acad. Sci. Paris 335 (2002), 577-580. Zbl 1013.05026 MR 1941297

[17] F. Hivert, J.-C. Novelli, and J.-Y. Thibon, The algebra of binary search trees. Theoret. Comput. Sci. 339 (2005), 129-165. Zbl 1072.05052 MR 2142078

[18] F. Hivert, J.-C. Novelli, and J.-Y. Thibon, Commutative combinatorial Hopf algebras. J. Algebraic Combin. 28 (2008), 65-95. Zbl 1181.16031 MR 2420780 
[19] D. Kreimer, Chen's iterated integral represents the operator product expansion. Adv. Theor. Math. Phys. 3 (1999), 627-670. Zbl 0971.81093 MR 1797019

[20] J.-L. Loday and M. O. Ronco, Hopf algebra of the planar binary trees. Adv. Math. 139 (1998), 293-309. Zbl 0926.16032 MR 1654173

[21] C. Malvenuto and C. Reutenauer, Duality between quasi-symmetric functions and the Solomon descent algebra. J. Algebra 177 (1995), 967-982.Zbl 0838.05100 MR 1358493

[22] F. Menous, J.-C. Novelli, and J.-Y. Thibon, Mould calculus, polyhedral cones, and characters of combinatorial Hopf algebras. Adv. Appl. Math. 51 (2013), 177-227. Zbl 06250895 MR 3071832

[23] J.-C. Novelli, F. Patras, and J.-Y. Thibon, Natural endomorphisms of quasi-shuffle Hopf algebras. Bull. Soc. Math. France 141 (2013), 107-130. Zbl 1266.05175 MR 3031674

[24] J.-C. Novelli and J.-Y. Thibon, Polynomial realizations of some trialgebras. In Proc. FPSAC 2006, San Diego 2006, 243-254.

[25] J.-C. Novelli and J.-Y. Thibon, Hopf algebras and dendriform structures arising from parking functions. Fund. Math. 193 (2007), 189-241. Zbl 1127.16033 MR 2289770

[26] J.-C. Novelli and J.-Y. Thibon, Noncommutative symmetric functions and Lagrange inversion. Adv. Appl. Math. 40 (2008), 8-35. Zbl 1133.05101 MR 2380843

[27] J.-C. Novelli, J.-Y. Thibon, and N. M. Thiéry, Algèbres de Hopf de graphes. C. R. Math. Acad. Sci. Paris 339 (2004), 607-610. Zbl 1062.16046 MR 2103196

[28] F. Patras and M. Schocker, Twisted descent algebras and the Solomon-Tits algebra. Adv. Math. 199 (2006), 151-184. Zbl 1154.16029 MR 2187402

Received December 6, 2011; revised February 9, 2012

L. Foissy, LMPA, Centre Universitaire de la Mi-Voix, 50, rue Ferdinand Buisson, 62228 Calais Cedex, France

E-mail: foissy@lmpa.univ-littoral.fr

J.-C. Novelli, Institut Gaspard Monge, Université Paris-Est Marne-la-Vallée,

5, Boulevard Descartes, Champs-sur-Marne, 77454 Marne-la-Vallée Cedex 2, France

E-mail: novelli@univ-mlv.fr

J.-Y. Thibon, Institut Gaspard Monge, Université Paris-Est Marne-la-Vallée,

5, Boulevard Descartes, Champs-sur-Marne, 77454 Marne-la-Vallée Cedex 2, France

E-mail: jyt@univ-mlv.fr 